\title{
ALEXANDRE PAPADIAMANTIS, UN SAINT PASSIONNÉ
}

\author{
Nicolas Evzonas
}

\section{L'Esprit du temps | Topique}

\section{$2012 / 3-n^{\circ} 120$ \\ pages 51 à 65}

\section{ISSN 0040-9375}

Article disponible en ligne à l'adresse:

http://www.cairn.info/revue-topique-2012-3-page-51.htm

Pour citer cet article :

Evzonas Nicolas, « Alexandre Papadiamantis, un saint passionné », Topique, $2012 / 3$ n 120 , p. 51-65. DOI : 10.3917/top. 120.0051

Distribution électronique Cairn.info pour L'Esprit du temps.

(c) L'Esprit du temps. Tous droits réservés pour tous pays.

La reproduction ou représentation de cet article, notamment par photocopie, n'est autorisée que dans les limites des conditions générales d'utilisation du site ou, le cas échéant, des conditions générales de la licence souscrite par votre établissement. Toute autre reproduction ou représentation, en tout ou partie, sous quelque forme et de quelque manière que ce soit, est interdite sauf accord préalable et écrit de l'éditeur, en dehors des cas prévus par la législation en vigueur en France. II est précisé que son stockage dans une base de données est également interdit. 


\title{
Alexandre Papadiamantis, un saint passionné
}

\author{
Nicolas Evzonas
}

Le chantre des passions : voilà le titre qui siérait véritablement à Alexandre Papadiamantis ${ }^{1}$ bien plus que celui de «Saint des Lettres grecques » que ce monstre sacré de la prose néo-hellénique doit à sa vie pieuse et austère ainsi qu'à sa propension à proclamer que tant qu'il vivrait, jamais il ne cesserait « de louer [s]on Christ avec vénération, de décrire amoureusement la nature et de dépeindre affectueusement les mœurs originales grecques ${ }^{2}{ }^{2}$. Si en dépit de son caractère résolument religieux, de son hellénocentrisme militant et de son fort ancrage rural, la littérature papadiamantienne continue à séduire un siècle et demi après sa naissance et à produire cette magie indéfinissable qui intrigue tant les critiques $^{3}$, c'est sans doute parce qu'elle sollicite puissamment notre pulsionnalité jusque dans ses couches les plus archaïques. «Au commencement était l'émotion » et non pas le Verbe, Céline nous le dit bien, et Papadiamantis excelle en effet à traduire en mots des agissements sensoriels et affectifs enfouis dans notre passé immémorial, conformément à la conception de l'écrivain «traduc-

1. Cet article s'inscrit dans le prolongement d'une thèse intitulée «Le Désir érotique dans l'œuvre d'Alexandre Papadiamantis », soutenue à 1'Université Paris-Sorbonne, en Janvier 2012.

2. Il s'agit d'un célèbre extrait du préambule de la nouvelle Chantre des Pâques

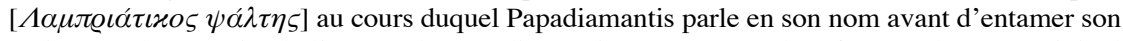
récit. Pour les extraits de l'œuvre de Papadiamantis, nous notons la référence du texte original $(O C$, II, p. 517) et ensuite la référence de la traduction utilisée. L'absence de deuxième référence indique que la traduction a été effectuée par nos soins. Pour les abréviations utilisées, cf. notre Bibliographie.

3. Voici quinze ans L. Proguidis évoquait déjà dans sa Conquête du roman (p. 52) plus de 25000 pages de gloses et depuis quinze ans une véritable «mode Papadiamantis » a fait exploser le nombre de travaux consacrés à son œuvre.

Topique, 2012, 120, 51-65. 
teur du monde sensible» selon Proust ou Beckett ${ }^{4}$. Si l'effervescence associée aux bases pulsionnelles de l'idiome natal échappe nécessairement au lecteur non hellénisant, tout comme une part de l'enchantement lié au style linguistique unique de Papadiamantis, lequel fait appel à un vocabulaire «palimpsestique» permettant de voyager dans toutes les périodes de l'histoire de la Grèce, tous les lecteurs profiteront pleinement de l'imaginaire foisonnant d'un auteur considéré comme le plus grand créateur de mythes de la littérature néo-hellénique ${ }^{5}$. D'ailleurs, la magie opère tout autant au sein des jeunes générations grecques pour qui la langue sui generis de Papadiamantis est difficilement accessible, presque une langue étrangère, ce qui témoigne des vertus transcendantes et translinguistiques de cette œuvre empreinte de passion, tant au sens étymologique de la souffrance (deuil lié à une perte) ${ }^{6}$, qu'au sens philosophique de l'accident (au-delà du principe de la volonté active $)^{7}$ et au sens économique de l'hyperinvestissement (caractère itératif, voire addictif de l'élan passionnel) ${ }^{8}$.

Ce texte se propose comme une exploration du dynamisme émotionnel de l'œuvre de Papadiamantis et, en même temps, comme une promenade initiatique dans un univers littéraire qui, malgré l'engouement suscité dans quelques cercles spécialisés, demeure largement méconnu en France ${ }^{9}$. Notre approche, qui sera forcément assez sommaire au vu de l'espace limité d'un article, privilégiera la psychanalyse en tant que grille de lecture et le lecteur en tant que sujet de signification du texte, ce qui veut dire que l'auteur en tant qu'entité empirique et psychobiographique à l'origine du texte ne nous intéressera pas. Bien évidemment, dès lors que nous explorons les productions d'un prosateur unique et que nous axons notre démarche sur le psychique, nous acceptons que nos analyses puissent éveiller une certaine curiosité relative au psychisme de l'écrivain. Malgré toutes les célébrations de la structure, l'auteur n'est jamais mort et même Roland Barthes, qui avait proféré sa sentence mortelle en plein iconoclasme soixante-huitard ${ }^{\mathbf{1 0}}$, a avoué peu après son « désir de l'auteur ${ }^{\mathbf{1 1}}$ » sans doute parce

4. Cf. J. Kristeva, «L'amour de 1'autre langue», p. 61-85, ainsi que l'ouvrage collectif sous la direction de C. Dumoulié et de M. Riaudel, le Corps et ses Traductions.

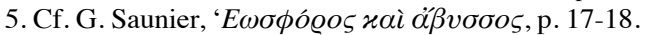

6. Passion vient du lat. passio < patior «supporter, souffrir» dérivé du grec $\pi \alpha \dot{\theta} \theta o \varsigma$ «émotion de l'âme, expérience subie, malheur» de la même famille étymologique que $\pi \varepsilon ́ v \theta o \varsigma$ «deuil», $\pi \varepsilon v \theta \varepsilon \dot{\varepsilon} \omega$ «pleurer un mort». Cf. Lewis and Short, A Latin Dictionnary, s.v. patior; P. Chantraine, Dictionnaire étymologique de la langue grecque, s.v. $\pi \alpha ́ \sigma \chi \omega$.

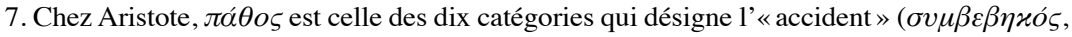
traduit par accidens par les scolastiques) consistant à subir une action. En conséquence, il renvoie à un état passif de l'âme (cf. TLFi, s.v. passion ; P. Chantraine, op . cit.).

8. Cf. S. de Mijolla-Mellor, le Besoin de croire, p. 102 sq.

9. Sur ce sujet, cf. E. Karagiannis-Moser, «Éléments pour la réception d'A. Papadiamantis en France». Il est par ailleurs intéressant pour le public français d'apprendre que Papadiamantis a suscité l'intérêt de la princesse Marie Bonaparte qui a visité sa maison à Skiathos et organisé en 1908 une fête en son honneur à Athènes.

10. Cf. son texte «La mort de l'auteur».

11. Cf. R. Barthes, le Plaisir du texte, p. 39. 
que « comme tout refoulé, l'auteur cherche toujours à revenir ${ }^{\mathbf{1 2}}$ »!

Notre réflexion s'articulera autour de quatre axes, quatre «passions », et notre démonstration se fera à partir de textes majeurs ou mineurs issus de toutes les périodes de la production artistique de Papadiamantis. Celle-ci s'étend de 1879 à 1911 (date du décès de l'écrivain) et comporte trois romans considérés comme historiques par la critique et près de cent quatre-vingts nouvelles dont trente possèdent pour cadre les quartiers populaires d'Athènes, les autres se déroulant dans les îles Sporades notamment à Skiathos, lieu de naissance de l'auteur.

\section{LA PASSION VOYEURISTE}

Toute l'œuvre de Papadiamantis est littéralement traversée de regards ${ }^{\mathbf{1 3}}$. D'innombrables occurrences d'expressions ou de termes relatifs à l'acte de voir inondent les textes et concernent tout un aréopage de situations et de personnages animés par une incoercible pulsion scopique. Celle-ci trouve son expression la plus pure dans l'observation clandestine qui s'impose dans les textes comme la modalité visuelle papadiamantienne par excellence, empreinte non pas de réalisme ou de «photographisme », comme se plaît à l'affirmer l'auteur lui-même, mais d'impressionnisme et d'hédonisme. Considérons de plus près quelques exemples caractéristiques.

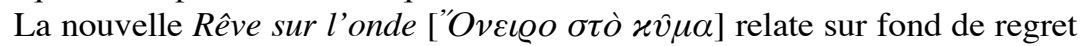
l'histoire d'un citadin qui vivait jadis en pleine nature dans une merveilleuse autarcie narcissique: «J'étais alors un bel adolescent, je voyais mon visage prématurément tanné par le soleil se refléter dans les ruisseaux et les fontaines, j'exerçais mon corps souple et élancé à escalader les rochers et les montagnes. ${ }^{\mathbf{1 4}}$ » Le bonheur de ce Narcisse bucolique consistait parmi d'autres à se sentir en osmose avec l'élément aquatique tel un fœtus bienheureux dans le ventre maternel: «J'éprouvais une douceur, un enchantement ineffables, je m'imaginais ne faire qu'un avec le flot, participer de sa nature, fraîche, amère, liquide. ${ }^{15}$ » Ce héros, qui ne connaissait d'autres délices que ceux que son propre corps lui procurait, a accédé lors d'une nuit initiatique, que nous avons tous éprouvée à un moment ou à un autre de notre existence en proie à la même fascination, au monde mystérieux de l'Autre. Après quantité de rationalisations vraisemblablement associées à l'éducation religieuse du héros qui servent à transformer le désir d'un voyeurisme actif en un voyeurisme passif et involontaire, ce qui ne

12. Nous paraphrasons J. Bellemin-Noël, Psychanalyse et Littérature, p. 143 : «Comme tout refoulé, 1'auteur cherche toujours à tourner la censure !»

13. Sur la prépondérance du visuel dans l'œuvre de Papadiamantis, cf. l'étude circonstanciée de R. Bouchet, le Guetteur invisible.

14. $O C$, III, p. $261 ; A N$, p. 19.

15. $O C$, III, p. $267 ; A N$, p. 27. 
peut que réactiver chez nous les astuces de notre curiosité sexuelle d'antan chargée de culpabilité, le jeune satyre des montagnes savoure en catimini le spectacle excitant d'une jouvencelle dénudée en train de prendre un bain de mer. La scène étant divisée en deux parties correspondant à deux visions différentes, la première de face, qui fait fuir, et l'autre de dos, qui enchante, elle s'impose à notre inconscient, qui n'a rien oublié de nos anciennes quêtes visant à percer l'énigme de nos origines, en tant que fantasme de castration consécutif à la découverte de la choquante réalité de la différence sexuelle. La castration transparaît dans le texte sous forme de «déni de réalité » [Verleugnung] aboutissant à la description d'une femme fantasmatique, onirique et poétique: «Je devinais sa poitrine, ses seins fermes et élégants, qui s'offraient aux haleines de la brise, au parfum divin de la mer. C'était un souffle, une image fantastique, un rêve qui flottait sur l'onde. C'était une néréide, une nymphe, une sirène, qui voguait comme un vaisseau magique, comme vogue la nef des songes... 16 ${ }_{\gg}$ Faut-il mentionner que l'autre sirène évoquée dans le corpus papadiamantien, l'enchanteresse qui rend fou un pirate dans la Fille de Bohême ['H Гvфтолоvं $\lambda \alpha$ ], n'est pas une femme-oiseau, comme on pourrait s'y attendre dans une digression aux influences homériques, mais une femme dotée d'une queue ichtyomorphe?

S'écartant de la fiction papadiamantienne classique de l' «observateur

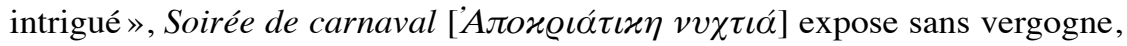
dans une version préhitchcockienne ${ }^{17}$ apte à flatter la scopophilie sulfureuse de notre Ça, le penchant d'un étudiant solitaire à l'espionnage de ses voisins depuis sa chambre donnant sur la cour d'une résidence collective. Le profil claustrophile et agoraphobe du héros est assez rapidement nuancé en gynophobie, tandis que son attirance pour une adolescente brune aux prémices de la féminité suggère sa peur plus spécifique de l'Autre sexué, peur que nous partageons tous à des degrés divers. Le jour le plus festif du carnaval, le héros refuse de participer aux soirées auxquelles il est convié sous prétexte qu'il ne sait pas danser et choisit de s'adonner à sa passion voyeuriste. Mais alors qu'il «danse involontairement dans son lit ${ }^{18}$ » sur l'air d'une chanson de carnaval grivoise, il songe que «même quand sa mère le berçait enfant, cela ne lui avait jamais procuré autant de volupté que ne le firent cette nuit-là les cris et les effusions de tous les voisins ${ }^{19}$ ». Ce bercement autoérotique, qui ranime en nous les premières expériences du holding nous solidarisant avec le personnage, peut cependant s'avérer une source de souffrance lorsqu'il demeure le moyen exclusif de se procurer du plaisir. Voilà pourquoi notre voyeur, qui conçoit sa vie comme «un long et triste carême ${ }^{20} »$, doit se consoler en pensant que «c'était lui le plus

16. $O C$, III, p. $269-270 ; A N$, p. 31.

17. Cf. le célèbre Fenêtre sur cour [Rear Window].

18. $O C$, II, p. 308.

19. $O C$, II, p. 313.

20. $O C$, II, p. 311. 
heureux, car sans assister à aucune fête, il participait à trois ou quatre divertissements simultanément ${ }^{21}$ » et que «sans doute faut-il, pour apprécier la musique et la danse, se tenir à distance; de près, le bruit assourdit et anéantit le jugement ${ }^{22}{ }$. Se souvenant de la fable d'Ésope, il murmure «ils sont trop verts ${ }^{23}$ ». Le récit papadiamantien nous incite à donner un nom au conflit à l'origine de ce type de construction mentale que nous fabriquons en vue de nous soustraire à notre défaillance : le «complexe du renard» (qui, contraint à renoncer aux grappes trop haut perchées, les dénigre).

\section{LA PASSION JALOUSE}

La distance qui maintient les héros papadiamantiens séparés de l'objet de leur désir et empêche la fusion corporelle est souvent présentée dans les textes comme la conséquence inévitable d'un échec amoureux dû à la présence maléfique d'un rival. Loin de constituer une simple cause de souffrance, ce terzo incommodo, qui accapare l'esprit des amants jusqu'à l'obsession, parvient à devenir pour ces derniers, par le déchaînement imaginatif qu'il déclenche, une source de plaisir hors pair pouvant s'apparenter, ne serait-ce que sous forme négative, à cette jouissance au-delà du phallus, transversale au sexe et au langage que Lacan appelle «Autre » ${ }^{24}$. On relèvera que chez Platon ${ }^{25}$ le mélange plaisir/déplaisir susceptible de s'accrôtre sans limites est considéré comme la quintessence de la passion jalouse.

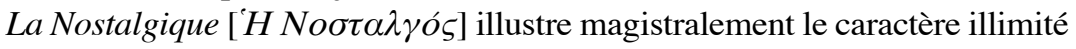
de ce pathos mixte qui plonge ses racines dans notre réservoir œedipien. L'amour d'un jeune insulaire pour sa voisine plus âgée ployant sous le joug d'un époux autoritaire, cet amour donc né sous les auspices du désir triangulaire prend véritablement son essor à partir du moment où l'héroïne, qui a pourtant accepté de s'enfuir nuitamment avec son soupirant pour regagner son village natal, repousse ses avances en lui révélant sans ambages son indisponibilité érotique. Le soupçon déchirant de l'existence d'un autre amant, le fantasme de jouer le rôle de «nocher des Enfers destiné à réunir deux êtres qui vont se livrer à des caresses infernales $\mathbf{2 6}^{\mathbf{2 6}}$, les baisers de l'autre qu'il traque sur les lèvres de la femme, les aveux coupables qu'il s'efforce d'arracher à celle qu'il croit désormais perfide, la confirmation délicieuse de ces fictions qu'il pense avoir obtenue,

21. $O C, \mathrm{II}, \mathrm{p} .308$.

22. $O C$, II, p. 308.

23. $O C$, II, p. 309.

24. Sur la jalousie en tant que pendant négatif de la jouissance de l'Autre, cf. C. DUMOULIÉ, le Désir, p. 153-155.

25. Philèbe, $47 \mathrm{c}-48 \mathrm{~b}$.

26. $O C$, III, p. 59. 
les scénarios d'assassinat au poignard et de noyade de la cruelle couronné par le suicide de l'esseulé, la séduisante image de leurs cadavres gisant au fond de la mer au clair de lune, l'excitation, la colère, l'humiliation, la rage, la fascination, toute l'incroyable richesse des réactions du héros dédaigné révèle les vertigineuses possibilités hédoniques de la frustration que l'auteur partage avec le lecteur dans un délire transférentiel. Lorsque, au final de la nouvelle, le héros se repaît de l'idée que son amour est enfin payé de retour tout en faisant défiler dans son esprit le mari-rival accompagné de ses sbires et les soupirants fantastiques de sa compagne de voyage, l'inconscient des lecteurs mâles reconnaît certainement l'image affriolante de la mère polygame ainsi que l'attirance homosexuelle pour le père qui leur avait autrefois servi de rempart protecteur lorsqu'ils s'étaient trouvés confrontés à la castration féminine et au manque consécutif relatif au désir. La Nostalgique montre avec force comment le jaloux théâtralise par tous les moyens la jouissance insaisissable de l'Autre, qui constitue en vérité la jouissance suprême de l'inceste dont nous avons tous rêvé en tant que petits Edipes.

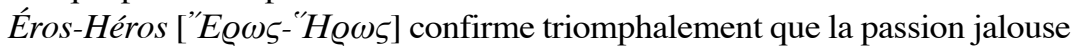
peut conduire bien « au-delà du principe de plaisir». Cette nouvelle met en scène un jeune marin qu'un sort ironique charge d'emmener en barque celle qu'il aime en secret depuis l'enfance jusqu'au domicile du vieillard qu'elle vient d'épouser. Plongé dans une infinie détresse et alors qu'il rame machinalement le visage livide tel Charon vers le séjour des morts, il se met à dévider voluptueusement dans son esprit des scénarios de vengeance : "D'un coup de pied, ou même moins encore, d'une simple pression d'un doigt de pied, il pouvait expédier sans frais trois âmes dans l'autre monde, l'époux, la belle-mère et l'épousée... si du moins il renonçait à sauver cette dernière ${ }^{27}$. » Le rival exécrable envié pour ses richesses matérielles «coulerait à pic comme une sonde, avec toutes ses maisons... ou plutôt sans ses maisons, sans ses terrains, sans ses domaines. ${ }^{28_{\text {» }}}$ La mère-mégère qui avait poussé sa fille à convoler «aurait à peine le temps de se signer pour la dernière fois, et ses cris de détresse seraient engloutis au fond de l'abîme. Le lendemain, au village, les prêtres prieraient pour le repos de son âme et inviteraient l'assistance à faire pénitence. Et pendant quarante jours, toutes les vieilles bigotes de la paroisse s'abstiendraient de manger des poissons, de crainte qu'ils n'aient effleuré le cadavre de la noyée. ${ }^{29} »$ Vivant pleinement son fantasme, le héros «fendait les flots comme un dauphin, soufflait et crachait des gerbes d'eau comme une baleine, lançait en avant son bras tranchant comme l'éperon d'un espadon. Il nageait avec le bras droit et serrait contre lui la jeune femme de son bras gauche. ${ }^{\mathbf{3 0}}$ » Et après un tel parcours glorieux sur les flots, les

27. $O C$, III, p. $180 ; A L$, p. $133-134$.

28. $O C$, III, p. 180 ; $A L$, p. 134.

29. $O C$, III, p. $180 ; A L$, p. $134-135$.

30. $O C$, III, p. 181 ; $A L$, p. 135. 
deux amoureux «se laisseraient tomber sur la sable, harassés, à moitié noyés, dégoulinants d'eau de mer. Créés et baptisés pour la seconde fois, nouvel Adam et nouvelle Ève, plus nus dans leurs vêtements mouillés leur collant à la peau que s'ils avaient été complètement nus. ${ }^{\mathbf{3 1}} \gg$ Magie de l'imagination : le drame d'un être parfaitement démuni se transforme en omnipotence mentale, à la façon de l'enfant en «désaide» [Hilfosigkeit] que nous avons autrefois été lorsque, en l'absence de notre mère, nous opposions à notre désemparement et à notre totale impuissance la solution radicale et inversée de la toute-puissance de la pensée, la satisfaction hallucinatoire de nos désirs.

Le familier des textes de Papadiamantis, qui sanctionnent toute débauche pulsionnelle par des épilogues factices d'assujettissement à la règle religieuse, ne s'étonnera guère de lire à l'extrême fin du récit que le jaloux furieux d'ÉrosHéros « réprima sa passion, s'apaisa, se recueillit, pleura, et se montra hérö̈que dans son amour, un amour chrétien, un amour pur, fait de tolérance et de charité. ${ }^{32}$ » Or le fait que cet évanouissement miraculeux de l'Éros agressif survient à la suite d'une vision figurant la mère du héros se lamentant et criant qu'il ne faut pas céder à la tentation de passer à l'acte, on comprend plutôt que « inondé » par cette mère regrettablement «trop vieille pour le mettre au berceau ou le prendre dans ses bras ${ }^{33}$ », le jeune marin devient incapable de toute revendication érotique, comme il l'a d'ailleurs toujours été par le passé. Quant au rival abhorré, «le rapace venu d'ailleurs ravir la colombe ${ }^{34}$ », il s'impose à notre esprit, nullement étranger à ce type de manœuvres projectives, comme le réceptacle dans lequel nous déversons notre ressentiment à chaque fois que nous ne supportons plus notre insuffisance et que notre image devient trop pénible à assumer.

\section{LA PASSION PYGMALIONIENNE}

Il existe certains moments exceptionnels dans le monde artistique de Papadiamantis où l'homme et la femme se rencontrent sans qu'aucune distance ne les sépare, aucun écran, aucun tiers intermédiaire. Pendant ces instants uniques où toute forme d'éloignement semble abolie, les héros, brisant leurs protocoles habituels, expriment ouvertement leur sensualité. Étant donné que ces moments d'érotisme «à proximité » se déroulent invariablement dans un contexte de danger et de risque qui livre la femme sans défense et sans connaissance au toucher de l'homme, nous pouvons parler d'une passion pygmalio-

31. OC, III, p. $181 ; A L$, p. 135.

32. $O C$, III, p. 182; $A L$, p. 136.

33. $O C$, III, p. 172; $A L$, p. 121.

34. $O C$, III, p. 179; $A L$, p. 132. 
nienne faisant référence au roi légendaire de Chypre qui s'éprend d'un objet inanimé ${ }^{35}$.

L'une des plus célèbres scènes de la littérature néo-hellénique est celle qui immortalise une telle passion dans Rêve sur l'onde. Le héros qui provoque par un souhait enfantin l'apparition d'un péril maritime, quoique perturbé par l'«efficacité » de sa pensée «animique», sort de sa cachette et court in extremis à la rescousse de celle qu'il convoitait secrètement mais n'avait jamais osé approcher auparavant: «Je vis ce corps ravissant se débattre sous l'eau, plus proche des profondeurs que de la surface, plus proche de la mort que de la vie: je plongeai, saisis la jeune fille contre moi, et remontai. Quand je l'eus enlacée de mon bras gauche, il me sembla sentir la tiédeur de son souffle sur ma joue. J'étais arrivé à temps, grâce à Dieu ! Pourtant, elle ne donnait manifestement aucun signe de vie... ${ }^{\mathbf{3 6}}$ » Le sauveur «altruiste», qui se lance dans une brasse vigoureuse sentant ses «forces redoubler comme par miracle» au contact du corps agonisant, comme s'il fallait neutraliser la femme afin de se sentir dynamisé et fortifié, avouera le bonheur ineffable de cette expérience transcendante laquelle se résume à un toucher épidermique qu'il opposera aux accouplements mus par «les instincts du loup et du chien»: «Je me rappellerai longtemps encore ce corps tendre et délicat de jeune fille pure que j'ai senti un jour sur moi, pendant un court moment de ma si vaine existence. Ce fut un songe, une illusion, un enchantement. Comment comparer aux étreintes égoïstes, aux perfides et bestiales amours de ce monde, cet exquis, ce sublime frôlement? Je n'avais pas saisi un fardeau ; j'avais trouvé le soulagement, le repos. Jamais je ne me suis senti aussi léger qu'aussi longtemps que j'ai porté le poids de son corps... J'étais l'homme qui avait un instant réussi à tenir un rêve entre ses mains, son propre rêve. ${ }^{37} \gg$ Ce rêve si poétiquement dépeint, qui laisse transparaître un besoin viscéral d'emprise et d'instrumentalisation de la femme jusqu'à l'annihilation de son altérité, n'est pas sans évoquer les œillères que nous nous imposons, lorsque cramponné à notre narcissisme afin d'éviter l'expérience douloureuse du manque de l'objet, nous nous obstinons à ne voir chez l'autre que cette partie limitée, cette «partialité » qui convient à nos fantasmes.

La vérité psychologique de cette passion pour la femme privée de ses sensations devient plus transparente dans la scène finale de la Fille de Bohême au cours de laquelle un violent séisme et une «heureuse catastrophe » permettent au jeune tzigane du roman épris de sa demi-sœur de laisser enfin agir son Éros: «Moron l'embrassait, la serrait contre son cœur et se sentait comblé. [...] Mais Aïma était sans connaissance et le jeune homme volait des étreintes, volait des caresses, volait des baisers. [...] Et cet instant, cet instant d'épouvante et

35. Cf. le «fantasme de Pygmalion» proposé comme une entité clinique à part entière par S. de Mijolla-Mellor dans son Choix de la sublimation, p. 260-277.

36. $O C$, III, p. 272 ; $A N$, p. 34.

37. $O C$, III, p. 272 ; $A N$, p. 34-35. 
d'angoisse où le monde s'écroulait et où régnait l'horreur, était un siècle de félicité. ${ }^{38} \gg$ Il n'est pas anodin que cette appropriation clandestine de l'intimité de l'autre succède à une contemplation également clandestine. Profitant de l'assoupissement de son aimée avant que le tremblement de terre ne le transforme en un opportun évanouissement, le héros, qui n'a pas cessé d'affirmer tout au long du roman son penchant à «voir sans être vu », observe sa belle aux yeux clos dans un état analogue à celui du narrateur proustien devant Albertine endormie : «Est-il en effet plus ravissant spectacle pour l'amant que celui de son aimée dormant comme un agneau, d'un sommeil serein et innocent, à la clarté opaline d'une lampe ? Est-il rien de plus doux pour lui que de suivre le rythme de sa respiration, est-il rien de plus enviable que de contempler les ondulations de sa poitrine, rien de plus enivrant que d'aspirer son souffle suave et d'admirer les perles de rosée de ses tempes, le diadème de boucles blondes posé sur son front? Moron, transporté de bonheur, buvait lentement, goutte à goutte, ce calice d'ivresse ${ }^{39}$. » Le lecteur, qui n'a pas oublié que la gitane Aïma arbore une chevelure d'ébène, a de quoi s'étonner devant cette crinière dorée que l'amoureux transi «voit » ${ }^{\mathbf{4 0}}$, à moins de s'identifier à ce dernier en reconnaissant que dans le secret de son âme lui aussi manipule l'autre, tel un fétiche, au gré de ses obsessions et conformément à ses agalmata.

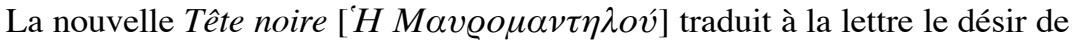
Pygmalion puisque le récit culmine avec l'enlacement sensuel d'un célibataire jusqu'alors étranger aux délices d'Aphrodite et une femme depuis longtemps changée en pierre : "Il alla l'embrasser, pour qu'une fois au moins se vérifie la sentence: "Une seule âme pour deux corps !" Ah ! Elle fut bien la seule, TêteNoire, cette âme de pierre, cette jeune fille qui n'avait connu ni l'émoi ni l'amour, cette nymphe parée d'huîtres et de coquillages, cette couche défaite, couverte de galets, cette veuve qui n'avait jamais eu de mari, [toujours vêtue de noir mais les yeux secs,] oui, elle fut bien la seule à offrir un asile au vieux marin tourmenté, la seule à recevoir les baisers et les étreintes de mon cousin

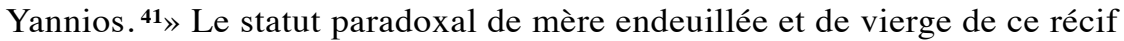
anthropomorphe, qui offre un asile au héros dans un moment de faiblesse où il risque de sombrer dans la mer, ne peut renvoyer qu'à la mère-madone de nos premières années, toujours vivante dans notre inconscient. On pourrait éventuellement imputer l'impossibilité du héros d'aller vers d'autres amantes à son lien indéfiniment prolongé avec cette mère-pierre figée dans l'immobilité du temps.

38. $O C$, I, p. $655 ; F B$, p. $402-403$; nous soulignons.

39. $O C$, I, p. $652-653 ; F B$, p. 399.

40. Sur cette blondeur incongrue dans l'extrait, cf. Ch. YAMPANI, Recherches sur «la Jeune Gitane » [la Fille de Bohême] de Papadiamantis.

41. $O C$, II, p. $166 ; A N$, p. 78. 


\section{LA PASSION GYNOCIDE}

Aux antipodes de sa réputation de «Saint des Lettres grecques », Papadiamantis est en vérité le saint serial killer des Lettres grecques ! Son univers littéraire généralement thanatophile est constellé de décès en apparence accidentels d'êtres féminins (fiancées, parturientes, fillettes), lorsqu'il ne s'agit pas de meurtres à peine ou pas du tout voilés, sans compter les assassinats métaphoriques et les innombrables crimes fantasmés. Véritable obsession structurelle de l'œuvre donc, cette dernière passion peut être nommée gynocide.

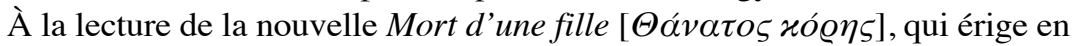
sujet principal le trépas d'une demoiselle s'apprêtant à convoler et explique que «ce que la défunte a reçu en échange de la trivialité du mariage et d'autres médiocrités du même acabit ne peut être que meilleur, car on ne peut imaginer pire $^{\mathbf{4 2}}$ », on peut ne voir qu' un banal plaidoyer contre l'hymen. Or quand la Fille de Bohême périt écrasée sous la statue d'Artémis quelques heures avant ses noces, l'on devine que le réel enjeu du décès prématuré, c'est la virginité de la

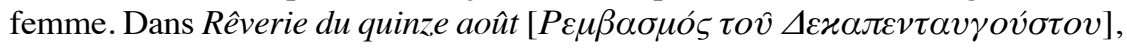
la disparition d'une jouvencelle à peine nubile «cueillie par la Sainte Vierge [...] avant d'être infectée par le contact avec les vanités du monde ${ }^{\mathbf{4 3}}{ }_{\text {» }}$ est susceptible de solliciter plus puissamment notre affectivité si notre moi émotif relève que la jeune fille a été conçue précisément à la date commémorant la fin terrestre de la Mère du Christ. Contamination de la vie par le germe de la mort donc. La scène primitive en action. «Avant-hier la fleur virginale a déployé ses pétales rouges. Hier elle est devenue épouse ; le jour suivant mère, accouchée, morte ${ }^{44} \gg$ : la maternité est ensanglantée et mortifère à l'inverse de celle, unique, de la Vierge

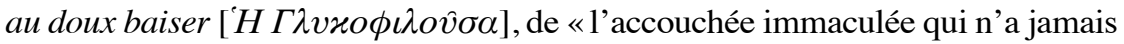
connu la couche d'un homme ${ }^{\mathbf{4 5}}$ » et qui représente de ce fait «l'expression la plus élevée de l'affection maternelle $\mathbf{4 6}^{\mathbf{4}}$. Catholique ou orthodoxe, croyant ou non croyant, on adule tous une mère qui ne s'est jamais impliquée dans l'acte sexuel, qui ne s'est jamais soumise à la violence du père, une mère entièrement et exclusivement dévouée à l'amour de ses rejetons. Les bénéfices psychologiques procurés aux lecteurs de l'œuvre papadiamantienne, inscrite sous le signe d'une mariologie superlative, transcendent la foi, la géographie ou la langue.

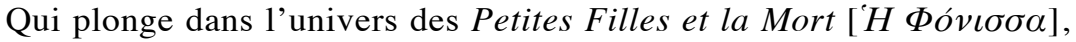
l'œuvre maîtresse de Papadiamantis, qui met explicitement en scène une campagnarde âgée tueuse «en série » de fillettes, s'expose à un véritable festival pulsionnel. L'action criminelle de cette matriarche d'une progéniture surabon-

42. $O C, \mathrm{IV}$, p. $190 ; A L$, p. 251.

43. $O C, \mathrm{IV}$, p. 90 ; nous soulignons.

44. $O C$, III, p. 78.

45. $O C$, III, p. 76.

46. $O C$, III, p. 75 . 
dante, révoltée contre la perspective d'un asservissement prolongé par chaque nouvelle naissance dans la famille, se dévoile progressivement comme un assassinat de la féminité dans son potentiel fécond et comme un acte extrême de prévention : la meurtrière fait ainsi grâce à ses victimes de l'avenir funeste de la maternité et de l'acte atroce qui les rendra mères en leur faisant répéter la faute tragique à l'origine de la vie. D'où cette rêverie sur une stérilité salvatrice aux accents schopenhaueriens : «Comme ils étaient heureux ces [moines] qui dès leur plus tendre jeunesse, et comme par une inspiration divine, avaient compris quelle était la meilleure action qu'ils pouvaient faire : de ne pas donner la vie à d'autres malheureux. ${ }^{47}$ »

Ce pessimisme est subtilement traversé par un idéalisme pédophile visant à maintenir les victimes à tout jamais dans l'enfance ou plutôt à arrêter leur évolution vers l'enfer (social, moral, sexuel) de la maturité. Il n'est pas anodin que les prénoms des deux fillettes cajolées mais épargnées par la meurtrière évoquent

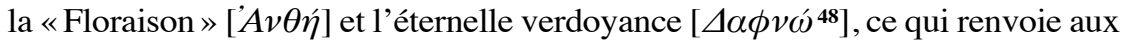
métaphores florales que l'héroïne utilise pour exprimer l'efflorescence regrettable de la féminité : «Krinio le petit lys, qui n'avait pas, tant s'en fallait, un teint de lys et de rose, bien que naturellement mince, montrait déjà des signes d'épanouissement. Comme elles grandissent, mon Dieu! pensait Francoyannou. Quel jardin, quelle prairie, quel printemps fait naître cette plante ? Et comme elle germe et pousse dru et déploie ses feuilles et se fortifie ! Et tous ces plants et ces jeunes pousses deviendront un jour des pelouses et des fourrés et des jardins? Et cela continuera donc à jamais ? ${ }^{49}$ » L'héroïne se charge donc, tel un ange exterminateur, de supprimer précocement, à la racine, la féminité susceptible d'éclore et de devenir un jour féconde.

Nos émotions souterraines réagissent intensément à la vision des fillettes câlinées à mort par la meurtrière qui, comme son nom de baptême l'indique

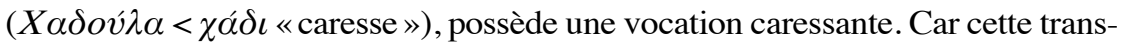
formation de l'embrassade maternelle en une étreinte asphyxiante nous fait revivre l'ancienne angoisse de suffocation par le sein délétère de notre génitrice «abjecte ${ }^{\mathbf{5 0}}$ ». Antidote à cette résurgence anxiogène, l'image éclatante de la vieille fille hommasse du récit, qui s'abstient du cercle infernal de l'hymen et de la reproduction et qui persévère en toute quiétude et en toute inoffensivité dans son autosuffisance androgynale. L'interchangeabilité d'attributs entre les

47. OC, III, p. 507 ; PFM, p. 170.

48. Le prénom fait, selon toute vraisemblance, référence au mythe de la nymphe antique Daphné dont les variantes les plus connues (celle de Parthénios de Nicée et celle d'Ovide) convergent sur l'aversion de cette dernière pour l'hymen et les mâles, et sur son sauvetage-transformation en laurier juste avant le moment fatidique de la perte de sa virginité.

49. $O C$, III, p. 433 ; PFM, p. 40.

50. Sur la notion de la mère abjecte (pas encore reconnue comme objet, mais ab-jet), cf. J. Kristeva, Pouvoirs de l'horreur. 
sexes et le mélange de genres chez quasiment tous les personnages des Petites Filles et la Mort, qui reprend en concentré un trait récurrent du microcosme littéraire de Papadiamantis, ne peut évidemment pas laisser indifférents les créatures tristement «sexionnées » que nous sommes, toujours nostalgiques de notre état fusionnel avec la mère à l'orée de notre existence. Lorsque Platon nous raconte par la voix d'Aristophane le mythe des êtres doubles scindés par Zeus, désespérés et qui n'aspirent qu'à se réunir et à se refondre dans leur ancienne unicité onaniste $^{51}$, il met en mots cette désirance universelle dont Papadiamantis traduit toute l'ambivalence affective à travers les esquisses inégales de ses héros androgynes.

La fin de la chronique de l'hérö̈ne infanticide nous incite à régresser encore plus loin dans le temps de notre ontogenèse. Poursuivie par les gendarmes et alors qu'elle s'efforce de traverser un étroit bras de mer pour aller se confesser dans une petite chapelle, la femme tourmentée, qui s'évertue en tuant à effacer les traumatismes du mariage et de la maternité consécutifs au traumatisme primordial de sa naissance, se laisse noyer d'une manière qui évoque un accouchement à l'envers. Comment alors ne pas la suivre dans ce retour aux sources [nostos], dans ce bain amniotique capable de nous faire oublier nos souffrances, de soigner les blessures de notre sexualité, de purifier l' «inter urinas et faeces» de notre façon d'aimer ${ }^{52}$ ? Ce voyage nost-algique (douleur du retour) n'est-il pas l'enjeu de la jouissance de l'écriture comme de la lecture?

\section{EN GUISE DE CONCLUSION}

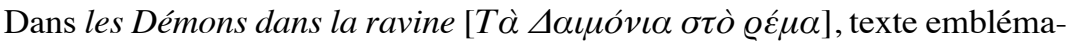
tique de l'univers personnel de Papadiamantis, le héros, qui a déserté un lieu sacré pour succomber à une tentation libidinale, avoue que cette expérience d'égarement est l'allégorie de toute sa vie en recourant à une citation de Dante: «Chè la diritta via era smarrita ${ }^{53}$ ». Si cette perte de la «dirrita via » peut être interprétée comme l'échec du perfectionnement métaphysique que l'auteur cherchait dans sa vie et comme le choix érotique d'un art foisonnant de passions tentatrices, elle résume à merveille le contenu et la structure d'une œuvre littéraire vouée au détournement et au déguisement, conditions capitales, selon l'optique freudienne ${ }^{54}$, pour le plaisir préliminaire (formel, esthétique) annonciateur d'une jouissance émanant de sources souterraines. L'ars poetica de cet

51. Banquet, $189 \mathrm{~d}-193 \mathrm{~d}$.

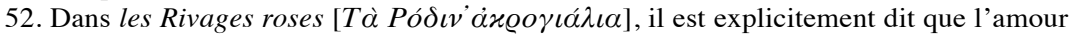
reproducteur est un «amour dans le fumier» $(O C, \mathrm{IV}, \mathrm{p} .142)$, ce qui ne va pas sans mobiliser l'ancien fantasme de notre naissance cloacale.

53. OC, III, p. 243.

54. Cf. S. Freud, «Personnages psychopathiques à la scène», p. 126-129. 
écrivain «national» de la Grèce réside peut-être avant tout dans sa capacité de satisfaire la perversité polymorphe de notre Ça tout en déjouant la vigilance de notre Cerbère surmoïque.

\author{
Nicolas EVZONAS \\ 36-38, rue Saint-Paul \\ 75004 Paris \\ nicolas.evzonas@gmail.com
}

\title{
BIBLIOGRAPHIE
}

\section{A. Papadiamantis}

BOUCHET, R., le Guetteur invisible : Étude du regard dans l'œuvre d'Alexandre Papadiamantis, Mém. de maîtrise, Paris, INALCO, 1983 (dactylographié).

Bouchet, R., le Nostalgique : l'Imaginaire de l'espace dans l'œeuvre d'Alexandre Papadiamantis, Paris, Presses de l'Univ. de Paris-Sorbonne, 2001.

Evzonas, N., le Désir érotique dans l'œuvre d'A. Papadiamantis, Th. de Doctorat, Université Paris-Sorbonne, 2012.

Karagiannis-Moser, E., «Éléments pour la réception d'A. Papadiamantis en France», Revue des Études Néo-helléniques (REN), IV (numéro spécial sur Papadiamantis), 1995, p. 159-201.

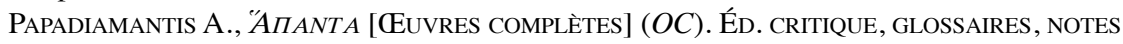
ET INDEX réalisés par N.D. Triantafyllopoulos, Athènes, Domos, 1981-1992 (6 vol.).

Papadiamantis A., l'Amour dans la neige (AN), trad. de R. Bouchet, Paris, Hatier, 1993.

PaPadiamantis A., Autour de la lagune et autres nouvelles $(A L)$, trad. de R. Bouchet, Carouge/Genève, Zoé, 2005.

Papadiamantis, A., la Fille de Bohême (FB), trad. de K. Coressis, Arles, Actes Sud/Athènes, Institut français d'Athènes, 1996.

Papadiamantis, A., les Petites Filles et la Mort (PFM), trad. de M. Saunier, Paris, Actes Sud, 1995.

Proguidis, L., la Conquête du roman : de Papadiamantis à Boccace, Paris, Les Belles Lettres, 1997.

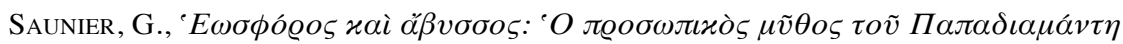
[Lucifer et l'Abîme: le Mythe personnel de Papadiamantis], Athènes, Éd. Agra, 2001.

YAMPANI, Ch., Recherches sur «la Jeune Gitane» [la Fille de Bohême] de Papadiamantis, Mém. de DEA, Paris, Université de Paris-Sorbonne, 1989 (dactylographié). 


\section{B. OUVRAGES THÉORIQUES ET GÉNÉRAUX}

ANDRÉ, S. et Gosselini, G., Qu'est-ce que la pédophilie?, Bruxelles, Luc Pire, 2008.

BARTHES, R., «La mort de l'auteur» (1968), in Essais critiques, t. 4, le Bruissement de la langue, Paris, Éd. du Seuil, 1993, p. 63-69.

Barthes, R., le Plaisir du texte (1973), Paris, Éd. du Seuil, 1982.

Bellemin-NoËL, J., Lire de tout son inconscient, Paris, PUV, 2011.

BELLEMIN-NoËL, Psychanalyse et Littérature, Paris, PUF, 2002.

Carloni, Gl. et Nobili,D., la Mauvaise Mère : Phénoménologie et Anthropologie de l'infanticide, Paris, Payot, 1977.

CAYAT, E. Et Fischetti, A., le Désir et la Putain : les Enjeux cachés de la sexualité masculine, Paris, Albin Michel, 2007.

Dumoulié, C., le Désir, Paris, Armand Colin, 1999.

Dumoulié, C. et Riaudel, M. (dir.), le Corps et ses Traductions, Paris, Éd. Desjonquères, 2008.

Freud, S., «Personnages psychopathiques à la scène » (1905 ou 1906), in Résultats, Idées, Problèmes, Paris, PUF, 1984.

Klein, M. et Riviere, J., l'Amour et la Haine, Paris, Payot, 1998.

KRISTEVA, J., «L'amour de l'autre langue » (1996), in l'Avenir d'une révolte, Paris, CalmannLevy, 1998, p. 61-85.

KristeVA, J., Pouvoirs de l'horreur : Essais sur l'abjection, Paris, Éd. du Seuil, 1980.

Mijolla-Mellor (de), S., le Besoin de croire, Paris, Dunod, 2004.

Mijolla-Mellor (de), S., le Choix de la sublimation, Paris, PUF, 2009.

Milner, M., On est prié de fermer les yeux : le Regard interdit, Paris, Gallimard, 1991.

Ovide, les Métamorphoses, Paris, Gallimard, 1992.

PARTHÉnios De NicÉe, Passions d'amour, éd. bilingue français-grec, Paris, Éd. Jérôme Million, 2008.

Platon, Banquet, éd. bilingue français-grec, Paris, Les Belles Lettres, 2010.

Platon, Philèbe, Paris, Flammarion, 2002.

SChopenhauer, A., Métaphysique de l'amour sexuel, Paris, Mille et une Nuits, 2008.

WARNER, M., Seule entre toutes les femmes : Mythe et Culte de la Vierge Marie, Marseille, Rivages, 1989.

\section{Nicolas Evzonas - Alexandros Papadiamantis, un saint passionné}

Résumé: En dépit du titre du «Saint des Lettres grecques», Alexandros Papadiamantis, reconnu comme l'écrivain «national » de la Grèce moderne, se dessine à travers ses textes comme le chantre des passions et le porte-parole d'une pulsionnalité exubérante. L'article propose une exploration du dynamisme émotionnel de son œuvre axée autour de quatre thématiques: le regard clandestin hédoniste, les scénarios jaloux théâtralisant la jouissance insaisissable de l'Autre, l'Éros pour la femme privée de ses sensations recelant la peur viscérale de l'altérité et la mort récurrente des êtres féminins assimilée à des meurtres préventifs au nom de la virginité et de la stérilité.

Mots-clés : Grèce - Papadiamantis - Voyeurisme - Jalousie - Jouissance de l'Autre Pygmalion - Femme inconsciente - Virginité - Tueur en série - Plaisir préliminaire. 
Nicolas Evzonas - Alexandros Papadiamantis, a Passionate Saint.

Abstract: Despite being referred to as a 'Saint of Greek Literature', Alexandros Papadiamantis, a writer of national acclaim in Modern Greece, produces texts which describe extraordinary passions and uncontrolled and uncontrollable drives. This article explores the emotional dynamics underpinning his work in the light of four themes: the covert Hedonistic gaze, scenarios involving jealousy, staging the ineffable jouissance of the Other, Eros for women deprived of physical sensation due to a deeply-rooted fear of otherness and the recurring deaths of feminine beings assimilated with preventive murder in the name of virginity and sterility.

Key-words: Greece - Papadiamantis - Voyeurism - Jealousy - Jouissance of the Other - Pygmalion - Unconscious Woman - Virginity - Serial Killer - Preliminary Pleasure. 